\title{
THE EXISTENCE AND NON-EXISTENCE OF POSITIVE SOLUTIONS OF ELLIPTIC SYSTEMS IN $R^{N}$
}

\author{
YUAN-WEI QI*
}

\begin{abstract}
In this paper, we study elliptic system $\Delta u+|x|^{\sigma_{1}} f(u, v)=0, \Delta v+|x|^{\sigma_{2}} g(u, v)=0$ and its quasilinear counterpart, where $x \in R^{n}, \sigma_{1}, \sigma_{2}>-2$. Under the assumption that there exists a Hamiltonian $H(u, v)$ such that $f(u, v)=H_{v}(u, v)$ and $g(u, v)=H_{u}(u, v)$, we establish a number of existence and non-existence results which was previously available mainly for the rather special case of $f=f(v)$ and $g=g(u)$ and $\sigma_{1}=\sigma_{2}=0$. The main tools are a priori estimates and a Pohozaev identity.
\end{abstract}

1. Introduction. In a previous paper (see [13]) we studied the elliptic system

$$
\begin{aligned}
& \triangle u+f(u, v)=0 \\
& \triangle v+g(u, v)=0
\end{aligned}
$$

and its quasilinear counterpart

$$
\begin{aligned}
& \operatorname{div}(A(\nabla u, \nabla v) \nabla u)+f(u, v)=0, \\
& \operatorname{div}(B(\nabla u, \nabla v) \nabla v)+g(u, v)=0
\end{aligned}
$$

in $R^{n}$, where $f$ and $g$ are $C^{1}$ functions with $f(u, 0)=g(0, v)=0, A(s, t)$ and $B(s, t)$ are functions which are positive and smooth for $s, t \in R^{n} \backslash\{0\}$. In particular, we established a number of results on existence and non-existence of ground state, mostly when $f(u, v)=f(v)$ and $g(u, v)=g(u)$. A ground state is a positive classical solution in $R^{n}$ which tends to zero at $\infty$.

The main purpose of present study is to extend those results to systems which take the form

$$
\begin{aligned}
& \Delta u+r^{\sigma_{1}} f(u, v)=0 \\
& \Delta v+r^{\sigma_{2}} g(u, v)=0
\end{aligned}
$$

and

$$
\begin{aligned}
& \operatorname{div}(A(\nabla u, \nabla v) \nabla u)+r^{\sigma_{1}} f(u, v)=0, \\
& \operatorname{div}(B(\nabla u, \nabla v) \nabla v)+r^{\sigma_{2}} g(u, v)=0,
\end{aligned}
$$

where $r=|x|, \sigma_{1}, \sigma_{2}>-2$ and $f, g$ will take the general form of $f(u, v)=H_{v}(u, v)$ and $g(u, v)=H_{u}(u, v)$. Here $H(u, v)$ is a $C^{2}$ function for $(u, v) \geq 0, H_{u}(u, v)$ and $H_{v}(u, v)$ represent the partial derivative of $H$ with respect to $u$ and $v$ respectively.

There have been extensive studies of system (1) for both boundary value problems in bounded domain as well as in $R^{n}$ in recent years. See [1], [2], [3], [5], [6], [7], [9], $[12],[14],[15],[16],[17]$ and [18] for some results of the recent developement.

The most important ingredients of the present study are a priori estimates and a generalized Pohozaev identity. In order to derive the desired a priori estimates we make the following

\section{Basic Assumption:}

$$
f(u, v) \geq C v^{p}, \quad g(u, v) \geq C u^{q} \quad \text { for }(u, v) \text { positive but close to zero, }
$$

*Department of Mathematics, Hong Kong University of Science \& Technology, Hong Kong (maqi@uxmail.ust.hk). 
where the constants $C, p, q$ satisfy $C>0, p, q \geq 1$ and $p q>1$.

The contents of this paper are as follows. In section 2 , we derive the essential a priori estimates under only the basic assumption and show that if $(p, q)$ satisfies $\max \left(2(p+1)+\sigma_{1}+p \sigma_{2}, 2(q+1)+q \sigma_{1}+\sigma_{2}\right) \geq(n-2)(p q-1)$, then there exists no positive solution $(u, v)$ of (1.1) in any exterior domain $r \geq a>0$ and tends to zero as $r \rightarrow \infty$, where $r=|x|$ is the radial variable in $R^{n}$ (see Theorem 1). Nevertheless, if $\max \left(2(p+1)+\sigma_{1}+p \sigma_{2}, 2(q+1)+q \sigma_{1}+\sigma_{2}\right)<(n-2)(p q-1)$, then (1.1) can admit positive radial solutions in the exterior domain $r \geq a>0$ for any $a>0$, as demonstrated by the explicit singular solution

$$
u=C_{1} r^{-\left[2(p+1)+\sigma_{1}+p \sigma_{2}\right] /(p q-1)}, \quad v=C_{2} r^{-\left[2(q+1)+q \sigma_{1}+\sigma_{2}\right] /(p q-1)} .
$$

of (1.1) when $f(u, v)=v^{p}, g(u, v)=u^{q}$, where $C_{1}$ and $C_{2}$ are some positive constants. In addition, we establish a Pohozaev identity when $f(u, v)=H_{v}(u, v), g(u, v)=$ $H_{u}(u, v)$ and $\sigma_{1}=\sigma_{2}$.

In section 3 , we explore further this special case and show that under mild assumptions of $H(u, v)$, there exists no positive radial solution of (1.1) in $R^{n}$ when $(p, q)$ is subcritical (see Definition 1 in section 3), which are the contents of Theorem 2 and Theorem 3. On the other hand, if $(p, q)$ is supercritical or critical, we show existence of positive radial solutions of (1.1) by a shooting argument in Theorem 4 and Theorem 5.

In section 4, non-existence results for various cases of (1.2) are demonstrated. A case worth to mention is that when $A=B=A\left(\left|u^{\prime} v^{\prime}\right|\right)$ for a radial solution, a much sharper result is obtained since we have a Pohozaev identity when $f(u, v)=H_{v}(u, v)$, $g(u, v)=H_{u}(u, v)$ and $\sigma_{1}=\sigma_{2}$.

A radial solution $(u(r), v(r))$, with $r=|x|$ of (1.1) satisfies the following system:

$$
\begin{array}{ll}
\left(r^{n-1} u^{\prime}\right)^{\prime}+r^{n-1+\sigma_{1}} f(u, v)=0, & \text { for } r>0, \\
\left(r^{n-1} v^{\prime}\right)^{\prime}+r^{n-1+\sigma_{2}} g(u, v)=0, & \text { for } r>0, \\
u^{\prime}(0)=0, \quad v^{\prime}(0)=0 . &
\end{array}
$$

The equations for radial solution of (1.2), under appropriate conditions of $A$ and $B$, are

$$
\begin{array}{ll}
\left(A\left(u^{\prime}, v^{\prime}\right) r^{n-1} u^{\prime}\right)^{\prime}+r^{n-1+\sigma_{1}} f(u, v)=0, & \text { for } r>0 \\
\left(B\left(u^{\prime}, v^{\prime}\right) r^{n-1} v^{\prime}\right)^{\prime}+r^{n-1+\sigma_{2}} g(u, v)=0, & \text { for } r>0 \\
u^{\prime}(0)=0, & v^{\prime}(0)=0 .
\end{array}
$$

We note by passing that in the detailed proof of many results, various constants will not be distinguished because the minor role they play as well as our concern to simplify the notation.

2. Asymptotic behaviour and preliminary results. The purpose of this section is twofolds. First, we state and prove some preliminary yet important results on asymptotic behaviour of positive radial solutions of semilinear system (1.1) on exterior domain $|x| \geq a>0$ which can be obtained using elementary analysis. These results culminate in giving an optimal asymptotic estimate in Lemma 2 which will be used later on to discuss existence and non-existence. A non-existence result as a direct consequence of Lemma 2 is proved in Theorem 1. Second, we derive a generalized Pohozaev type identity which is more general than the one we obtained in [13] for the case of $f(u, v)=f(v), g(u, v)=g(v)$ and $\sigma_{1}=\sigma_{2}=0$. 
Lemma 1. Suppose $f(u, v) \geq 0, g(u, v) \geq 0$ for $(u, v)$ positive but sufficiently small, then there exist no positive radial solutions $(u, v)$ of (1.1) defined for $r>a>0$ which tend to zero as $|x| \rightarrow \infty$ if $n \leq 2$. Furthermore, if $n>2$ and $(u, v)$ is a positive radial solutions of (1.1) defined for $r>a$ and tends to zero as $r \rightarrow \infty$, then

$$
-u^{\prime} \geq C r^{1-n}, \quad u \geq C r^{2-n}, \quad-v^{\prime} \geq C r^{1-n}, \quad v \geq C r^{2-n}
$$

for all $r$ sufficiently large, where $C>0$ is a positive constant. In addition, for all $r$ sufficiently large,

$$
r u^{\prime}+(n-2) u>0, \quad r v^{\prime}+(n-2) v>0 .
$$

Proof. See [2] and [13].

In what follows a more accurate asymptotics will be given using the basic assumption of this paper.

Lemma 2. Suppose $f(u, v) \geq C v^{p}, g(u, v) \geq C u^{q}$ for $(u, v)>0$ but small, $(u, v)$ is a positive radial solution of (1.1) defined for $r>a$ and tends to zero as $r \rightarrow \infty$. Then, the following asymptotic estimates hold:

$$
u=O\left(r^{-\left[2(p+1)+\sigma_{1}+p \sigma_{2}\right] /(p q-1)}\right), \quad v=O\left(r^{-\left[2(q+1)+q \sigma_{1}+\sigma_{2}\right] /(p q-1)}\right),
$$

$$
\left|u^{\prime}\right|=O\left(r^{-\left[2(p+1)+\sigma_{1}+p \sigma_{2}\right] /(p q-1)-1}\right), \quad\left|v^{\prime}\right|=O\left(r^{-\left[2(q+1)+q \sigma_{1}+\sigma_{2}\right] /(p q-1)-1}\right),
$$

as $r \rightarrow \infty$.

Proof. Suppose $r_{0}>a$ is large enough so that

$$
f(u, v) \geq C v^{p}, \quad g(u, v) \geq C u^{q}, u^{\prime}<0, \quad v^{\prime}<0
$$

on $\left[r_{0}, \infty\right)$, where $C$ is a positive constant. Integrating the first equation of (1.3) gives

$$
\begin{aligned}
& r^{n-1} u^{\prime}(r)-r_{0}^{n-1} u^{\prime}\left(r_{0}\right)=-\int_{r_{0}}^{r} s^{n-1+\sigma_{1}} f(u, v) d s \leq-C \int_{r_{0}}^{r} s^{n-1+\sigma_{1}} v^{p}(s) d s \\
& \leq-C v^{p}(r) \int_{r_{0}}^{r} s^{n-1+\sigma_{1}} d s \leq-\frac{C}{n+\sigma_{1}}\left(r^{n+\sigma_{1}} v^{p}(r)-r_{0}^{n+\sigma_{1}} v^{p}\left(r_{0}\right)\right) .
\end{aligned}
$$

Noting that $u^{\prime}\left(r_{0}\right)<0$, it follows that

$$
-u^{\prime}(r) \geq \frac{C}{n}\left(r^{1+\sigma_{1}} v^{p}(r)-r^{1-n} r_{0}^{n+\sigma_{1}} v^{p}\left(r_{0}\right)\right) .
$$

But, by Lemma $1,-u^{\prime}(r)>C r^{1-n}$ if $r$ is large. Hence,

$$
-u^{\prime}(r) \geq C r^{1+\sigma_{1}} v^{p}(r)
$$

if $r$ is sufficiently large. Integrating (2.4) from $r$ to $\infty$ one finds

$$
u(r)>\int_{r}^{\infty} s^{1+\sigma_{1}} v^{p} d s>C \int_{r}^{2 r} s^{1+\sigma_{1}} v^{p}(s) d s>C v^{p}(2 r) r^{2+\sigma_{1}} .
$$


Hence, combining the above inequality with the fact that $r^{n-2} v$ is an increasing function for $r \gg 1$ from Lemma 1 , one gets $u(r)>C v^{p}(r) r^{2+\sigma_{1}}$. Similarly, $v(r)>$ $C u^{q}(r) r^{2+\sigma_{2}}$. A combination of the above two inequalities yields that (2.2) holds. In turn, it implies (2.3) is valid when combined with Lemma 1.

\section{Corollary 1. Suppose $n \geq 3$ and}

$$
\max \left(2(p+1)+\sigma_{1}+p \sigma_{2}, 2(q+1)+q \sigma_{1}+\sigma_{2}\right) \geq(n-2)(p q-1) .
$$

Then there exists no positive radial solution of

$$
\left(r^{n-1} u^{\prime}\right)^{\prime}+r^{n-1+\sigma_{1}} f(u, v) \leq 0, \quad\left(r^{n-1} v^{\prime}\right)^{\prime}+r^{n-1+\sigma_{2}} g(u, v) \leq 0,
$$

which tends to zero as $r \rightarrow \infty$ on $[a, \infty)$ for any $a>0$ if the conditions of Lemma 2 on $f(u, v)$ and $g(u, v)$ are satisfied.

Proof. It is similar to the case of $\sigma_{1}=\sigma_{2}=0$, see [13].

REMARK. We note that (1.1) has a singular solution when $\max \left(2(p+1)+\sigma_{1}+\right.$ $\left.p \sigma_{2}, 2(q+1)+q \sigma_{1}+\sigma_{2}\right)<(n-2)(p q-1)$ which has the exact decaying rate as in Lemma 2.

Theorem 1. Suppose $f(u, v) \geq C v^{p}, g(u, v) \geq C u^{q}$ for $(u, v)>0$ but small, $n \geq 3$ and (2.5) holds. Then there exists nopositive solution of (1.1) which tends to zero as $|x| \rightarrow \infty$ in an exterior domain $|x|>$ a for any $a>0$.

Proof. Suppose by contradiction that there exists such a solution $(u, v)$. Let $r_{0}>a$ be so taken that $f(u(x), v(x)) \geq C v^{p}(x), g(u(x), v(x)) \geq C u^{q}(x)$ for all $x \in R^{n} \backslash B_{r_{0}}$, where $B_{r_{0}}$ is the ball with radius $r_{0}$ which centered at origin. Let

$$
\bar{u}(r)=\frac{1}{\omega_{n}} \int_{S^{n-1}} u(r, \theta) d s, \bar{v}(r)=\frac{1}{\omega_{n}} \int_{S^{n-1}} v(r, \theta) d s, \quad r>a,
$$

where $S^{n-1}$ is the unit sphere in $R^{n}, d s$ is the (n-1)-dimensional area element of $S^{n-1}$ and $\omega_{n}$ is the area of $S^{n-1}$. Then,

$$
\frac{1}{\omega_{n}} \int_{S^{n-1}} f(u, v) d s \geq \frac{C}{\omega_{n}} \int_{S^{n-1}} v^{p} d s \geq C \bar{v}^{p}(r), \frac{1}{\omega_{n}} \int_{S^{n-1}} g(u, v) d s \geq C \bar{u}^{q}(r)
$$

for $r>r_{0}$ by Jensen's inequality. Consequently, $(\bar{u}, \bar{v})$ is a solution of (2.6) for $r>r_{0}$. But this is impossible by Lemmas 1, 2 and Corollary 1.

In the rest of this section we shall restrict our attention to the narrow case of

$$
f(u, v)=H_{v}(u, v), \quad g(u, v)=H_{u}(u, v) \quad \text { and } \sigma_{1}=\sigma_{2}=\sigma
$$

where $H=H(u, v)$ is a $C^{2}$ function of $(u, v) \in R^{2}$ with $H(0,0)=0$. We begin by deriving a generalized Pohozaev identity of (1.1). It is clear that if $f(u, v)=f(v)$ and $g(u, v)=g(u)$, we can simply take $H(u, v)=F(v)+G(u)$, where $F(v)=\int_{0}^{v} f(s) d s$ and $G(u)=\int_{0}^{u} g(s) d s$.

Proposition 1. Let $(u, v)$. be positive on $\left[R_{1}, R_{2}\right)$, where $0 \leq R_{1}<R_{2} \leq \infty$. Suppose it is a solution of the following system

$$
\begin{aligned}
u^{\prime \prime}+\frac{n-1}{r} u^{\prime}+r^{\sigma} H_{v} & =0 \\
v^{\prime \prime}+\frac{n-1}{r} v^{\prime}+r^{\sigma} H_{u} & =0
\end{aligned}
$$


for $r \in\left[R_{1}, R_{2}\right)$. Then,

$$
E=r^{n}\left(u^{\prime} v^{\prime}+r^{\sigma} H(u, v)+\frac{a v u^{\prime}}{r}+\frac{b u v^{\prime}}{r}\right)
$$

satisfies

$$
\frac{d E(r)}{d r}=r^{n-1}\left[(a+b+2-n) u^{\prime} v^{\prime}+r^{\sigma}\left((n+\sigma) H-a v H_{v}-b u H_{u}\right)\right]
$$

for $r \in\left[R_{1}, R_{2}\right)$. Here $a$ and $b$ are two constants.

Proof. It is a simple exercise and we omit it.

3. Existence and non-existence theorems. In this section, we assume $f(u, v), g(u, v)$ and $\left(\sigma_{1}, \sigma_{2}\right)$ are as specified in (2.7). For this special case of $\sigma_{1}=\sigma_{2}=\sigma$, we may define what we mean a pair $(p, q)$ is subcritical or supercritical for system (1.1) in parallel with the case of $\sigma_{1}=\sigma_{2}=0$.

Definition 1. A pair $(p, q)$ with $p, q \geq 1$ and $p q>1$ is called subcritical if

$$
\frac{p+1}{p q-1}+\frac{q+1}{p q-1}>\frac{n-2}{2+\sigma}
$$

whereas the above inequality is reversed, it is called supercritical. If $(p, q)$ is so given that the equality holds in (3.1), it is said to be critical.

Theorem 2. Let $n>2, \sigma_{1}=\sigma_{2}=\sigma$, where $\sigma>-2$. Suppose that $f(u, v)>0$, $g(u, v)>0$ for all $u, v>0$ and moreover,

$$
f(u, v) \geq C v^{p}, \quad g(u, v) \geq C u^{q}, \quad H(u, v) \leq C\left(u^{q *+1}+v^{p *+1}\right)
$$

for all sufficiently small $u$ and $v$, where $p, p *, q, q *$ are constants binding the following inequalities

$$
\frac{p q-1}{(2+\sigma)(p+1)}(n+\sigma)-1<q * \leq q, \quad \frac{p q-1}{(2+\sigma)(q+1)}(n+\sigma)-1<p * \leq p .
$$

Assume further that there exists $(\alpha, \beta)$ which is not supercritical such that

$$
\frac{1}{\alpha+1} v H_{v}(u, v)+\frac{1}{\beta+1} u H_{u}(u, v) \leq H(u, v)
$$

for all $u>0$ and $v>0$, then there does not exist any positive radial solution of equation (1.1) in $R^{n}$.

REMARK. The assumption that $p, q, p *$ and $q *$ satisfy the relations given in (3.3) implies that $(p, q)$ is subcritical. In addition, $p * \leq \alpha, q * \leq \beta$.

Proof of Theorem 2. It is clear that any positive radial solution $(u, v)$ satisfies $u^{\prime}(r)<0, v^{\prime}(r)<0$ for $r>0$ and $(u, v)$ tends to zero as $r \rightarrow \infty$. Suppose to the contrary that there exists a radial positive solution. Thus, let $a=(n+\sigma) /(\alpha+1)$, $b=(n+\sigma) /(\beta+1)$ in the identity $(2.9)$,

$$
a+b+2-n \geq 0, \quad(n+\sigma) H-a v H_{v}-b u H_{u} \geq 0
$$


by (3.1) and (3.4). Consequently, one has, after an integration,

$$
r^{n}\left(u^{\prime} v^{\prime}+r^{\sigma} H(u, v)+\frac{a v u^{\prime}}{r}+\frac{b u v^{\prime}}{r}\right) \geq C^{2}
$$

for sufficiently large $r$, where $C>0$ (see Proposition 2 in [13]). Consequently, $r^{n}\left(u^{\prime} v^{\prime}+\right.$ $\left.r^{\sigma} H(u, v)\right) \geq C^{2}$. By (3.2) and Lemma 2, one gets that if $r$ is sufficiently large,

$$
\begin{aligned}
H(u, v) & \leq C\left(v^{p *+1}+u^{q *+1}\right) \\
& \leq C\left(r^{-(2+\sigma)(p *+1)(q+1) /(p q-1)}+r^{-(2+\sigma)(q *+1)(p+1) /(p q-1)}\right) .
\end{aligned}
$$

Hence, by (3.3),

$$
H(u, v)=o\left(r^{-n-\sigma}\right) \quad \text { as } r \rightarrow \infty .
$$

It then follows that $u^{\prime} v^{\prime} \geq C^{2} r^{-n}$ for $r \gg 1$. But, from $r u^{\prime}+(n-2) u>0, r v^{\prime}+(n-$ 2) $v>0$, one deduces $u^{\prime} v^{\prime} \leq C r^{-2} u v$. Thus, $u v \geq C r^{2-n}$. But, on the other hand, one gets using the estimates in Lemma 2 ,

$$
u \leq C r^{-(2+\sigma)(p+1) /(p q-1)}, \quad v \leq C r^{-(2+\sigma)(q+1) /(p q-1)} .
$$

Therefore, it must be true that

$$
\frac{(2+\sigma)(p+1)}{p q-1}+\frac{(2+\sigma)(q+1)}{p q-1} \leq n-2 .
$$

But this is in clear contradiction with our hypothesis that $(p, q)$ is subcritical. Hence, no positive radial solution exists for $(1)$ if $(p, q)$ is subcritical.

REMARK. The conclusion of Theorem 2 is true for a wide class of nonlinear functions $f(u, v)$ and $g(u, v)$. In particular if $f(u, v)=\lambda u+\lambda_{1} v+v^{p}, g(u, v)=$ $\lambda v+\lambda_{2} u+u^{q}$, where the three constants $\lambda, \lambda_{1}$ and $\lambda_{2}$ are non-negative, and $(p, q)$ is subcritical, then there exists no positive radial solution $(u, v)$ to $(1.1)$ in $R^{n}$. In addition, we note the condition $H(u, v) \leq C\left(u^{q *+1}+v^{p *+1}\right)$ with $(p *, q *)$ bound by (3.3) can be weakened substantially to guarantee the validity of Theorem 2 . For example, if

$$
H(u, v)=C_{1} \frac{v^{p+1}}{p+1}+C_{2} \frac{u^{q+1}}{q+1}+\sum_{i=3}^{N} C_{i} v^{p_{i}} u^{q_{i}}
$$

with $(p, q)$ subcritical and $\left(p_{i}, q_{i}\right)$ satisfying $(2+\sigma)\left[p_{i}(q+1)+q_{i}(p+1)\right]>(n+\sigma)(p q-1)$, $i=3,4, \ldots, N$, then there exists no radial ground state provided

$$
\bar{p}=\max _{3 \leq i \leq N} p_{i}, \quad \bar{q}=\max _{3 \leq i \leq N} q_{i}
$$

is a pair not supercritical.

Another way in which this theorem can be extended is to drop the upper bound assumption on $H(u, v)$, and estimate the decay of $H(u(r), v(r))$ as a function of $r$ from that of $f(u, v)$ and $g(u, v)$ under some monotonicity assumption on $f(u, v)$ and $g(u, v)$. The following is a result in this direction.

ThEOREM 3. Let $n>2, \sigma_{1}=\sigma_{2}=\sigma$, where $\sigma>-2$. Suppose that $f(u, v)>0$, $g(u, v)>0$ for all $u, v>0, f(u, v)$ and $g(u, v)$ are monotone increasing in $(u, v)$, and

$$
f(u, v) \geq C v^{p}, \quad g(u, v) \geq C u^{q}
$$


for all sufficiently small $u$ and $v$, where $(p, q)$ is subcritical. Assume further that there exists $(\alpha, \beta)$ which is not supercritical such that (3.4) holds for all $u>0$ and $v>0$, then there exists no positive radial solution of equation (1.1) in $R^{n}$.

Proof. It is clear from the proof of Theorem 2 that the only place $H(u, v) \leq$ $C\left(u^{q *+1}+v^{p *+1}\right)$ with $(p *, q *)$ satisfying (3.3) is used is to show (3.6) holds if $(u, v)$ is a ground state solution. So, we shall show that (3.6) is valid under our assumptions on $f(u, v)$ and $g(u, v)$. The rest will follow exactly as in Theorem 2 .

Choose $r_{0}>0$ sufficiently large such that $f(u(r), v(r))$ is a monotone decreasing function of $r$ for all $r \geq r_{0}$ and $-u^{\prime}(r) \geq C r^{1-n}$ (Lemma 1). This is possible since by our hypothesis $f(u, v)$ is monotone increasing in $(u, v)$ for $(u, v)>0$ small and $(u(r), v(r))$ is decreasing in $r$. Following the same procedure as in Lemma 2, we get

$$
\begin{aligned}
& -r^{n-1} u^{\prime}(r)+r_{0}^{n-1} u^{\prime}\left(r_{0}\right)=\int_{r_{0}}^{r} s^{n-1+\sigma} f(u, v) d s \\
& \geq f(u(r), v(r)) \int_{r_{0}}^{r} s^{n-1+\sigma} d s \geq \frac{f(u(r), v(r))}{n+\sigma}\left(r^{n+\sigma}-r_{0}^{n+\sigma}\right) .
\end{aligned}
$$

Using the fact that $u^{\prime}\left(r_{0}\right)<0$ and $-u^{\prime}(r) \geq C r^{1-n}$, we have

$$
-u^{\prime}(r) \geq C r^{1+\sigma} f(u(r), v(r)) .
$$

Consequently, by (2.3),

$$
f(u(r), v(r)) \leq C r^{-(2+\sigma)-(2+\sigma)(p+1) /(p q-1)} .
$$

Similarly,

$$
g(u(r), v(r)) \leq C r^{-(2+\sigma)-(2+\sigma)(p+1) /(p q-1)} .
$$

Hence,

$$
\begin{aligned}
H(u(r), v(r)) & =\int_{0}^{u(r)} g(s, 0) d s+\int_{0}^{v(r)} f(u(r), s) d s \\
& \leq f(u(r), v(r)) v(r)+g(u(r), v(r)) u(r) \leq C r^{-(2+\sigma)-(2+\sigma)(p+q+2) /(p q-1)}
\end{aligned}
$$

by (2.2). This, when combined with our hypothesis that $(p, q)$ is subcritical, shows (3.6) is valid.

Then, by repeating the same argument as in Theorem 2, we establish the theorem.

We now turn our attention to prove the existence of ground state solutions when $f$ and $g$ exhibit supercritical nonlinear growth when $u$ and $v$ are small. and

Proposition 2. Suppose $H_{u}>0$ and $H_{v}>0$ for $0<u<u_{0}$ and for $0<v<v_{0}$,

$$
\begin{aligned}
& (n+\sigma) H(u, v)-a v H_{v}(u, v)-b u H_{u}(u, v) \leq 0, \quad 0<u<u_{0}, 0<v<v_{0}, \\
& n-2-a-b \geq 0
\end{aligned}
$$

for some positive constants $a, b, u_{0}$ and $v_{0}$. Then there exists no radial solution to the following boundary value problem

$$
(B V P)\left\{\begin{array}{l}
\triangle u+H_{v}(u, v)=0, x \in B \\
\triangle v+H_{u}(u, v)=0, x \in B \\
0<u<u_{0}, 0<v<v_{0} \quad \text { in } B \text { and } u=v=0 \quad \text { on } \partial B
\end{array}\right.
$$


where $B=B_{R}$ is a ball centered at origin with radius $R>0$.

REMARK. The above result is a special case of a more general identity proved for all solutions of (BVP), not necessarily radial ones in [12] by Peletier and Van Der Vorst. For related results, see [17].

TheOREM 4. Suppose $f$ and $g$ satisfy, for $0<u<M, 0<v<M, f(u, v) \geq 0$, $g(u, v) \geq 0$ and there exists a $C^{2}$ function $H^{1}$ such that

$$
\begin{array}{lll}
f(u, v)=c_{1} v^{p}+H_{v}^{1}(u, v), & \text { with } & \limsup _{(u, v) \rightarrow 0} \frac{H_{v}^{1}(u, v)}{v^{p}}=0, \\
g(u, v)=c_{2} u^{q}+H_{u}^{1}(u, v), & \text { with } & \limsup _{(u, v) \rightarrow 0} \frac{H_{u}^{1}(u, v)}{u^{q}}=0,
\end{array}
$$

where $M, c_{1}$, and $c_{2}$ positive constants. Then there exists an infinite number of radial ground state solution of $(1)$ if $(p, q)$ is supercritical.

Proof. Apparently, $u^{\prime}(r)<0, v^{\prime}(r)<0$ for $r>0$ before the product $u v$ reaches zero. Let $u_{0}>0$ and $v_{0}>0$ be sufficiently small such that the conditions (3.7) in Proposition 2 are satisfied with $a$ close to $(n+\sigma) /(p+1)$ and $b$ close to $(n+\sigma) /(q+1)$. This is possible because of the assumption that $(p, q)$ is supercritical and $f(u, v)$ and $g(u, v)$ are small perturbation of $c_{1} v^{p}$ and $c_{2} u^{q}$ respectively. Consequently, there exists no radial solution of the boundary value problem (BVP) with initial value $0<u(0)<u_{0}, 0<v(0)<v_{0}$ for any $R>0$. Define

$$
A=\left\{(u(0), v(0)) \mid 0<u(0)<u_{0}, 0<v(0)<v_{0}\right\}
$$

which has the property that there exists $R>0$ such that the corresponding solution $(u, v)$ satisfies $v(r)>0, v^{\prime}(r)<0$ and $u^{\prime}(r)<0$ in $(0, R], u(r)>0$ for $0<r<R$ but $u(R)=0$. Similarly, let

$$
B=\left\{(u(0), v(0)) \mid 0<u(0)<u_{0}, 0<v(0)<v_{0}\right\}
$$

such that for the corresponding solution $(u, v)$ there exists $R>0$ with $u(r)>0$, $u^{\prime}(r)<0$ and $v^{\prime}(r)<0$ in $(0, R], v(r)>0$ for $0<r<R$ but $v(R)=0$. It is clear from Lemmas 3 and 4 in [13] that $A$ and $B$ are non-empty. In addition, they are both open by the classical theory of continuous dependence of solutions on initial values. Let $L$ be a straight line segment starting from a point in $A$ and ending at a point in $B$. It is clear that there exists a point $P=(\alpha, \beta)>0$ on $L$ which does not belong to either $A$ or $B$. Therefore, there are only two possibilities: (i) the corresponding solution with initial values $u(0)=\alpha, v(0)=\beta$ is a solution of the boundary value problem or (ii) it is a ground state solution. But, the first case is impossible. Hence, the corresponding solution is a ground state solution.

By taking different lines $L$ with different end points from $A$ and $B$, we can obtain an infinite number of ground state solutions.

Another case where there exists a a ground state solution is when

$$
\begin{aligned}
& f(u, v)=A_{1} v^{p}+\sum_{i=1}^{M} c_{i} v^{p_{i} *}+\sum_{j=1}^{N} d_{j}\left(p_{j}+1\right) v^{p_{j}} u^{q_{j}+1} \\
& g(u, v)=A_{2} u^{q}+\sum_{i=1}^{M} e_{i} u^{q_{i} *}+\sum_{j=1}^{N} d_{j}\left(q_{j}+1\right) v^{p_{j}+1} u^{q_{j}}
\end{aligned}
$$


where $(p, q)$ is critical, $p_{i} *>p, q_{i} *>q, i=1,2, \ldots N, p_{j} \geq p$ and $q_{j} \geq q, j=1,2, \ldots N$. Here $A_{1}, A_{2}, c_{i}, e_{i}$ and $d_{j}$ are positive constants.

In this case,

$H(u, v)=\frac{A_{1}}{p+1} v^{p+1}+\frac{A_{2}}{q+1} u^{q+1}+\sum_{i=1}^{M} c_{i} \frac{v^{p_{i} *+1}}{p_{i} *+1}+\sum_{i=1}^{M} e_{i} \frac{u^{q_{i} *+1}}{q_{i} *+1}+\sum_{j=1}^{N} d_{j} v^{p_{j}+1} u^{q_{j}+1}$.

Theorem 5. Suppose $f$ and $g$ are as given in (3.12) with $(p, q)$ critical. Then there exists an infinite number of radial ground state solutions.

Proof. It is clear that the conditions (3.7) in Proposition 2 are satisfied with $a=(n+\sigma) /(p+1)$ and $b=(n+\sigma) /(q+1)$. In particular, since $(p, q)$ is critical, $a+b=n-2$. The rest follows the same line of argument as in Theorem 4 . We invite the reader to fill the details.

4. Non-existence theorems for quasilinear systems. In this section we shall consider the special cases of quasilinear system (1.2) where for a radial solution $(u, v), A=A\left(h_{1}\right)$ is a $C^{1}$ function of a single variable $h_{1}$, in the range of $h_{1}>0$ and it is bounded around $h_{1}=0$, and $B=B\left(h_{2}\right)$ is a $C^{1}$ function of a single variable $h_{2}$ in the range of $h_{2}>0$ and is bounded around $h_{2}=0$. Here $h_{1}$ and $h_{2}$ maybe different combinations of $u^{\prime}$ and $v^{\prime}$. The primary difficulty is to get estimates as those given in (2.2) in Lemma 2.

LEMMA 3. Suppose all conditions of Lemma 2 on $f(u, v)$ and $g(u, v)$ are satisfied, and $(u, v)$ is a positive radial solution of (1.2) defined for $r>a$ and tends to zero as $r \rightarrow \infty$. Assume in addition that $A\left(u^{\prime}(r), v^{\prime}(r)\right)+B\left(u^{\prime}(r), v^{\prime}(r)\right) \leq M$ for all $r>a>0$. Then, for any $\epsilon>0$,

$$
u=o\left(r^{-\left[2(p+1)+\sigma_{1}+p \sigma_{2}\right] /(p q-1)+\epsilon}\right), \quad v=o\left(r^{-\left[2(q+1)+q \sigma_{1}+\sigma_{2}\right] /(p q-1)+\epsilon}\right)
$$

as $r \rightarrow \infty$.

Proof. It is similar to the case of $\sigma_{1}=\sigma_{2}=0$, see [13].

Given the situation as it is, the natural thing to do is to show that under various different forms of $A$ and $B, A\left(u^{\prime}, v^{\prime}\right)$ and $B\left(u^{\prime}, v^{\prime}\right)$ are bounded in $[a, \infty)$ for a positive radial solution $(u, v)$ of $(1.2)$ in $r \geq a$. We cite the following result which is obtained in [13]:

LEMMA 4. Let $f(u, v), g(u, v)$ satisfy the conditions of Lemma 1. Suppose $u(r)$ and $v(r)$ are positive radial solutions of (1.2) for $r \geq a>0$, which tend to zero as $r \rightarrow$ $\infty$, then $A\left(u^{\prime}, v^{\prime}\right)$ and $B\left(u^{\prime}, v^{\prime}\right)$ are bounded on $[a, \infty)$ provided $A$ and $B$ satisfy one of the following conditions: [(i)] $A=B=\Omega\left(\left|u^{\prime}\right|+\left|v^{\prime}\right|\right)$; [(ii)] $A=B=\Omega\left(\left|u^{\prime} v^{\prime}\right|\right)$; [(iii)] $A=A\left(\left|u^{\prime}(r)\right|\right), B=A\left(\left|u^{\prime}(r)\right|\right)$ or $B=B\left(\left|v^{\prime}(r)\right|\right)$.

Proof. See the proof of Lemmas 5 and 6 in [13].

Theorem 6. Suppose $A$ and $B$ in (1.2) satisfy one of the following conditions when $(u, v)$ is a radial solution: [(a)] $A=A\left(\left|u^{\prime}\right|\right), B=A\left(\left|u^{\prime}\right|\right)$ or $B=B\left(\left|v^{\prime}\right|\right)$; [(b)] $A=B=\Omega\left(\left|u^{\prime}\right|+\left|v^{\prime}\right|\right) ;[(\mathbf{c})] A=B=\Omega\left(\left|u^{\prime} v^{\prime}\right|\right)$.

Assume in addition that $A(h)$ and $B(h)$ are $C^{1}$ functions for $h>0$ and are bounded around $h=0$. Then for any positive radial solution $(u, v)$ of (1.2) which is defined for $r \geq a>0$ and tends to zero as $r \rightarrow \infty$, the following results hold: 
(i) Suppose $f(u, v) \geq 0, g(u, v) \geq 0$ for $(u, v)$ near 0 . Then,

$$
u \geq C r^{2-n}, \quad u^{\prime} \leq-C r^{1-n}, \quad v \geq C r^{2-n}, \quad v^{\prime} \leq-C r^{1-n}
$$

for all $r$ sufficiently large. Consequently no such solution is possible if $n \leq 2$.

(ii) Suppose $f(u, v) \geq C v^{p}, g(u, v) \geq C u^{q}$ and both of them are increasing function of $(u, v)$ for $(u, v)$ near 0 . Then, the estimates in (4.1) hold.

(iii) Suppose $n>2$ and $\max \left(2(p+1)+\sigma_{1}+p \sigma_{2}, 2(q+1)+q \sigma_{1}+\sigma_{2}\right)>(n-2)(p q-1)$. Then there exists no positive radial solution of (1.2) on $[a, \infty)$ for any $a>0$ if the conditions on $f$ and $g$ in (ii) are satisfied.

Proof. The proof of (i) is essentially same as in Lemma 1. (ii) is shown in Lemma 3. (iii) follows directly from (i) and (ii).

In the rest of this section we shall concentrate on the particular case where $A=B$ and $A=A\left(\left|u^{\prime} v^{\prime}\right|\right)$. In addition, the hypothesis (2.7) on $f(u, v), g(u, v)$ and $\sigma_{1}, \sigma_{2}$ is in force. The purpose is to derive a non-existence result comparable to that of Theorem 2 which is stronger than the above theorem. First, a generalized version of the identity in Proposition 1 is in order for the quasilinear system under consideration.

Proposition 3. Let $(u, v)$ be positive decreasing functions on $[0, \infty)$. Suppose

$$
\begin{aligned}
& \left(r^{n-1} u^{\prime} A\left(\left|u^{\prime} v^{\prime}\right|\right)\right)^{\prime}+r^{n-1+\sigma} f(u, v)=0 \\
& \left(r^{n-1} v^{\prime} A\left(\left|u^{\prime} v^{\prime}\right|\right)\right)^{\prime}+r^{n-1+\sigma} g(u, v)=0
\end{aligned}
$$

for $r \in\left(R_{1}, R_{2}\right)$, where $0 \leq R_{1}<R_{2} \leq \infty$. Then,

$$
E(r)=r^{n}\left(\int_{0}^{h} D(\rho) d \rho+r^{\sigma} H(u, v)+A\left(\left|u^{\prime} v^{\prime}\right|\right) \frac{a v u^{\prime}}{r}+A\left(\left|u^{\prime} v^{\prime}\right|\right) \frac{b u v^{\prime}}{r}\right)
$$

satisfies

$$
\begin{aligned}
\frac{d E(r)}{d r}= & r^{n-1}\left[(a+b+2-2 n) A\left(\left|u^{\prime} v^{\prime}\right|\right) u^{\prime} v^{\prime}+n \int_{0}^{h} D(\rho) d \rho\right. \\
& \left.r^{\sigma}((n+\sigma) H(u, v)-a v f(u, v)-b u g(u, v))\right] .
\end{aligned}
$$

Here $a$ and $b$ are two constants, $h=u^{\prime} v^{\prime}$ and $D(h)=A(h)+2 h A^{\prime}(h)$.

Proof. The calculation is tedious and we omit it.

Theorem 7. Let $n>2, \sigma_{1}=\sigma_{2}=\sigma$, where $\sigma>-2$. Suppose that $A(\nabla u, \nabla v)=B(\nabla u, \nabla v)=A(|\nabla u \cdot \nabla v|)$ and $A(h)$ is positive for all $h>0$ and bounded for $h$ close to zero. Assume also that $f(u, v)>0, g(u, v)>0$ for all $u, v>0$ and they satisfy the conditions (3.2) and (3.3) of Theorem 2. At last assume that

$$
\frac{1}{\alpha+1} v f(u, v)+\frac{1}{\beta+1} u g(u, v) \leq H(u, v)
$$

for all $u>0$ and $v>0$, where $(\alpha, \beta)$ satisfies

$$
\frac{n+\sigma}{\alpha+1}+\frac{n+\sigma}{\beta+1} \geq n d-2
$$


and $2 / n<d<2$ is a constant such that

$$
\int_{0}^{h} A(\rho) d \rho \leq d h A(h)
$$

Then there does not exist any positive radial solution of (1.2) in $R^{n}$.

REMARK. If $A_{h}=d A / d h \geq 0$, we can obviously take $d=1$ and condition (4.5) then reduces to the case of semilinear system in Theorem 1 . As in the case of Theorem 2 , there is no need to consider the case $(2+\sigma) \max (p+1, q+1)>(n-2)(p q-1)$ since it is covered in Theorem 6 . On the other hand, by letting $u=0$ and by an integration of (4.4) we get that $H(0, v) \geq C v^{\alpha+1}$ for $v>0$ small. Similarly, we get $H(u, 0) \geq C u^{\beta+1}$ for $u>0$ small. Hence, using (3.2) and (3.3) we find

$$
\alpha \geq q^{*} \geq \frac{2+\sigma}{n-2}, \quad \beta \geq p^{*} \geq \frac{2+\sigma}{n-2}
$$

when $(2+\sigma) \max (p+1, q+1) \leq(n-2)(p q-1)$. Consequently,

$$
\frac{n+\sigma}{\alpha+1}+\frac{n+\sigma}{\beta+1} \leq 2(n-2) \text {. }
$$

Thus, it follows from (4.5) that it is only for values $d \leq 2(n-1) / n$ that Theorem 7 provide a stronger result than that of Theorem 6 .

Proof of Theorem 7. Suppose to the contrary that there exists a positive radial solution of (1.2) in $R^{n}$. Then, as in Theorem 1 , such a solution must tend to zero as $r \rightarrow \infty$. Since $D(h)=A+2 h A^{\prime}(h)$, it follows that

$$
\int_{0}^{h} D(\rho) d \rho=2 h A(h)-\int_{0}^{h} A(\rho) d \rho \geq(2-d) h A(h) .
$$

Combining the above inequality with the result of Proposition 3, we obtain

$$
\begin{aligned}
(2-2 n+a+b) A\left(\left|u^{\prime} v^{\prime}\right|\right)+n \int_{0}^{h} D(\rho) d \rho & \geq(2-2 n+a+b+2 n-n d) A\left(\left|u^{\prime} v^{\prime}\right|\right) \\
& =(2+a+b-n d) A\left(\left|u^{\prime} v^{\prime}\right|\right) \geq 0 .
\end{aligned}
$$

In addition, $(n+\sigma) H(u, v)-a v f(u, v)-b u g(u, v) \geq 0$ by our hypothesis on $f(u, v)$, $g(u, v)$ and the choice of $a=(n+\sigma) /(\alpha+1), b=(n+\sigma) /(\beta+1)$. Consequently,

$$
r^{n}\left[\int_{0}^{h} D(\rho) d \rho+r^{\sigma} H(u, v)+A\left(\left|u^{\prime} v^{\prime}\right|\right) \frac{a v u^{\prime}}{r}+A\left(\left|u^{\prime} v^{\prime}\right|\right) \frac{b u v^{\prime}}{r}\right] \geq C^{2}
$$

for all $r$ sufficiently large, where $h=u^{\prime} v^{\prime}$, and $C$ is a positive constant. A detailed demonstration of the fact that $C$ is positive is exact like that of Proposition 2 and is omitted. From our previous discussion on the asymptotic behaviour of positive solutions it follows that $\left(u^{\prime}, v^{\prime}\right) \rightarrow 0$ as $r \rightarrow \infty$. Consequently using the boundedness of $A(h)$ near $h=0$, we have that for some $M>0$,

$$
\int_{0}^{h} D(\rho) d \rho=2 h A(h)-\int_{0}^{h} A(\rho) d \rho \leq 2 h A(h) \leq M h
$$


for $h$ sufficiently small. Using the above inequality and (4.7) we find that for $r$ sufficiently large, $r^{n}\left[M u^{\prime} v^{\prime}+r^{\sigma} H(u, v)\right] \geq C^{2}$. Nonetheless, argue as in Theorem 2, but this time using (4.1) instead of (2.2), we get $H(u, v)=o\left(r^{-n-\sigma}\right) \quad$ as $r \rightarrow \infty$. It then follows that $u^{\prime} v^{\prime} \geq C^{2} r^{-n}$ for $r \gg 1$. Hence, for any $\mu$,

$$
-u^{\prime}(r) r^{\mu}-v^{\prime}(r) r^{-\mu} \geq C r^{-n / 2}
$$

for $r \gg 1$. Clearly, $\mu$ can be chosen appropriately to satisfy $u(r) r^{\mu-1+n / 2} \rightarrow 0$, $v(r) r^{-\mu-1+n / 2} \rightarrow 0$ and $r^{-1} \int_{0}^{r} u(s) s^{\mu-1+n / 2} \rightarrow 0, r^{-1} \int_{0}^{r} v(s) s^{-\mu-1+n / 2} \rightarrow 0$ as $r \rightarrow \infty$, by the fact $(p, q)$ is subcritical and by the estimates in (4.1). But, an integration of of (4.8) would yield otherwise. Thus, we reach a contradiction.

\section{REFERENCES}

[1] BIRINDElli AND E. MitidiERI, Liouville theorems for elliptic inequalities and applications, Proceedings of Royal Society of Edinburgh, Ser. A., 128 (1998), pp. 1217-1247.

[2] P. Clement and Manasevitch and E. Mitidieri, Positive solutions for a quasilinear systems via blow up, Communication in Partial Differential Equations, 18 (1993), pp. 2071-2106.

[3] P. Clement and R. C. A. M. VAn Der Vorst, On a semilinear elliptic system, Diff. \& Int. Eq., 8 (1991), pp. 1317-1329.

[4] D.G. DE FiguriREdo AND P.L. Lions AND R. NusSBaum, A priori estimates and existence of positive solutions of semi-linear elliptic equations, J. Math. Pures et Appl., 61 (1982), pp. 41-63.

[5] M. García-Huidobro, R. MANÁsevich, E. Mitidieri ANd C. YARUR, Existence and nonexistence of positive singular solutions for a class of semilinear elliptic systems, Arch. Rational Mech. Anal., 140 (1997), pp. 253-284.

[6] J. Hulshof And R. C. A. M. VAn Der Vorst, Asymptotic behaviour of ground states, Proc. Amer. Math. Soc., 124 (1996), pp. 2423-2431.

[7] E. Mitidieri AND S. I. PohozAev, The absence of global positive solutions to quasilinear elliptic inequalities, Doklady Mathematics, 57 (1998), pp. 250-253.

[8] E. Mitidieri and G. Sweer and R. C. A. M. VAn Der Vorst, Nonexistence theorems for systems of quasilinear differential equations, Differential and Integral Equations, 8 (1995), pp. 1331-1354.

[9] E. MITIDIERI, A Rellich type identity and applications, Communications in Partial Differential Equations, 18 (1993), pp. 125-151.

[10] E. MITIDIERI, Non-existence of positive solutions of semilinear elliptic systems in $R^{n}$, Quaderno Matematico, 285 (1992).

[11] WeI-Ming Ni AND J. SERRIN, Existence and Non-existence theorems for quasi-linear partial differential equations. The anomalous case, Accas. Naz. Lincei, Convegni Dei Lincei, 77 (1986), pp. 231-257.

[12] L. A. PElETIER AND R. C. A. M. VAN DER VORST, Existence and non-existence of positive solutions of non-linear elliptic systems and the biharmonic equation, Differential and Integral Equations, 5 (1992), pp. 747-767.

[13] Y. W. QI, The existence and non-existence theorems of ground states of nonlinear systems, Communications in Partial Differential Equations, 23 (1998), pp. 1749-1780.

[14] J. SERrin AND H. Zou, Non-existence of positive solutions of Lane-Emden systems, Differential and integral equations, 9 (1996), pp. 635-653.

[15] J. SERRIN AND H. ZOU, Existence of positive entire solutions of elliptic Hamiltonian systems, Communications in Partial Differential Equations, 23 (1998), pp. 577-599.

[16] M. VERON AND T. RAOUX, Asymptotics of solutions of some nonlinear elliptic systems, Communications in Partial Differential Equations, 21 (1996), pp. 1035-1086.

[17] R. C. A. M. VAN DER VORST, Variational identities and applications to differential systems, Arch. Rational Mech. Anal., 116 (1991), pp. 375-398.

[18] H. Zou, Existence and non-existence of positive solutions of the scalar field systems in $R^{n}, I$, Calculus of Variations, 4 (1996), pp. 219-248. 